\title{
PROTOPLANETARY DISK MASSES IN THE YOUNG NGC 2024 CLUSTER
}

\author{
Rita K. Mann ${ }^{1}$, Sean M. Andrews ${ }^{2}$, Josh A. Eisner ${ }^{3}$, Jonathan P. Williams ${ }^{4}$, Michael R. Meyer ${ }^{5}$, \\ James Di Francesco ${ }^{1,6}$, John M. CARPEnter ${ }^{7}$, And Doug Johnstone ${ }^{1,6}$ \\ ${ }^{1}$ National Research Council Canada, 5071 West Saanich Road, Victoria, BC, V9E 2E7, Canada; rita.mann@nrc-cnrc.gc.ca \\ ${ }^{2}$ Harvard-Smithsonian Center for Astrophysics, 60 Garden Street, Cambridge, MA 02138, USA \\ ${ }^{3}$ Steward Observatory, University of Arizona, 933 North Cherry Avenue, Tucson, AZ 85721, USA \\ ${ }^{4}$ Institute for Astronomy, University of Hawaii, 2680 Woodlawn Drive, Honolulu, HI 96822, USA \\ ${ }^{5}$ ETH Zürich, Institute for Astronomy, Wolfgang-Pauli-Strasse 27, 8093, Zurich, Switzerland \\ ${ }^{6}$ Department of Physics and Astronomy, University of Victoria, Victoria, BC, V8P 1A1, Canada \\ ${ }^{7}$ Department of Astronomy, California Institute of Technology, MC 249-17, Pasadena, CA 91125, USA \\ Received 2014 October 6; accepted 2015 January 26; published 2015 March 24
}

\begin{abstract}
We present the results from a Submillimeter Array survey of the $887 \mu \mathrm{m}$ continuum emission from the protoplanetary disks around 95 young stars in the young cluster NGC 2024. Emission was detected from 22 infrared sources, with flux densities from $\sim 5$ to $330 \mathrm{mJy}$; upper limits (at $3 \sigma$ ) for the other 73 sources range from 3 to $24 \mathrm{mJy}$. For standard assumptions, the corresponding disk masses range from $\sim 0.003$ to $0.2 M_{\odot}$, with upper limits at $0.002-0.01 M_{\odot}$. The NGC 2024 sample has a slightly more populated tail at the high end of its disk mass distribution compared to other clusters, but without more information on the nature of the sample hosts it remains unclear if this difference is statistically significant or a superficial selection effect. Unlike in the Orion Trapezium, there is no evidence for a disk mass dependence on the (projected) separation from the massive star IRS $2 \mathrm{~b}$ in the NGC 2024 cluster. We suggest that this is due to either the cluster youth or a comparatively weaker photoionizing radiation field.
\end{abstract}

Key words: circumstellar matter - protoplanetary disks - stars: formation - stars: pre-main sequence

\section{INTRODUCTION}

The fundamental properties of circumstellar disks play a critical role in the formation and evolution of planets. While detailed knowledge of disk properties has come from extensive studies of nearby associations like Taurus-Auriga and $\rho$ Ophiuchus (e.g., Beckwith et al. 1990; Osterloh \& Beckwith 1995; Andrews \& Williams 2005, 2007; Andrews et al. 2009, 2010), stars in these regions form in loose agglomerations and are generally unaffected by their external environment. The majority of stars in the galaxy, including the Sun, formed in densely populated rich clusters (Lada \& Lada 2003; Porras et al. 2003; Williams 2010). The high stellar density and ultraviolet radiation from nearby massive stars in these regions can affect disk properties, threatening their development and potentially limiting their lifespans (Bonnell et al. 2003; Johnstone et al. 1998). Probing disk evolution in rich clusters is therefore crucial to our understanding of planet formation.

The Orion star-forming complex contains the nearest rich clusters with massive stars, and is arguably the best region for studying how disk properties are affected by their environment. It is home to the clusters NGC 2024 ( $\sim 0.5$ Myr; Meyer 1996; Ali et al. 1998; Levine et al. 2006) and the Orion Nebula Cluster (ONC, 1-2 Myr; da Rio et al. 2010; Reggiani et al. 2011). Near-infrared observations of the NGC 2024 cluster members have revealed 233 young stars (Meyer 1996), of which $\gtrsim 85 \%$ exhibit an infrared excess indicative of warm dust in the inner regions of protoplanetary disks (Haisch et al. 2000, 2001). These stars are still deeply embedded in molecular cloud material (Barnes et al. 1989; Lada et al. 1991), in line with their suggested extreme youth. The most massive star in the region is thought to be IRS $2 \mathrm{~b}$, with a spectral type in the range of O8-B2 (Barnes et al. 1989; Bik et al. 2003). Although the earlier end of that range could more easily explain the radio continuum emission in the region, it has been suggested that a collection of slightly lower mass stars could together be responsible for the total ionizing flux (Meyer et al. 2008). IRS $2 \mathrm{~b}$ is located $5^{\prime \prime}$ northwest of the early B-type star IRS 2 (Grasdalen 1974), the brightest infrared and radio source in NGC 2024 (Barnes et al. 1989; Rodríguez et al. 2003).

The relative youth of NGC 2024 makes it a particularly appealing region to probe the initial properties of disks. Through comparisons with the older ONC (and similar clusters, e.g., $\sigma$ Ori; Williams et al. 2014), we can constrain key timescales for disk evolution in rich clusters. With reference to pre-main sequence evolution models, the positions of the NGC 2024 members in a color-magnitude diagram indicate very young ages: the Baraffe et al. (1998) models suggest < 1 Myr, and the D'Antona \& Mazzitelli (1997) models argue for $\sim 0.5 \mathrm{Myr}$ (Meyer 1996; Ali et al. 1998; Levine et al. 2006). The absolute ages of young stars are highly uncertain (e.g., Soderblom et al. 2014). However, in a relative sense these are significantly younger ages than have been estimated for other clusters using the same technique and models (e.g., see Eisner \& Carpenter 2003). They overlap with the earliest stages of the evolution process for circumstellar material (e.g., Evans 2009).

Although the infrared excess emission found for most NGC 2024 members confirms the ubiquity of disks in this region, it traces only a small fraction of the disk material. The low optical depths for the continuum emission at longer, (sub)millimeter wavelengths is required to quantitatively probe the masses of these disks. While the disk population in the neighboring ONC has now been studied in some detail at (sub)millimeter wavelengths with interferometers (Williams et al. 2005; Eisner \& Carpenter 2006; Eisner et al. 2008; Mann \& Williams 2010; Mann et al. 2014), the disks in NGC 2024 are relatively 
Table 1

Summary of Submillimeter Array Observations

\begin{tabular}{|c|c|c|c|c|c|c|c|}
\hline $\begin{array}{l}\text { Field } \\
\text { (1) }\end{array}$ & $\begin{array}{c}\alpha(\mathrm{J} 2000) \\
(2)\end{array}$ & $\begin{array}{c}\delta(\mathrm{J} 2000) \\
(3)\end{array}$ & $\begin{array}{c}\text { UT Date } \\
(4)\end{array}$ & $\begin{array}{c}\tau \\
(5)\end{array}$ & $\begin{array}{c}\sigma(\mathrm{mJy} / \text { beam }) \\
(6)\end{array}$ & $\begin{array}{c}\theta_{b}\left({ }^{\prime \prime}\right) \\
(7)\end{array}$ & $\begin{array}{l}\mathrm{PA}_{b}\left(^{\circ}\right) \\
(8)\end{array}$ \\
\hline A & 054147.0 & -015447 & 2011 Sep 23 & $0.06-0.07$ & 1.0 & $2.5 \times 1.5$ & 158 \\
\hline B & 054145.4 & -015418 & 2011 Sep 23 & $0.06-0.07$ & 3.0 & $2.5 \times 1.5$ & 158 \\
\hline $\mathrm{C}$ & 054145.1 & -015454 & 2011 Sep 23 & $0.06-0.07$ & 3.5 & $2.5 \times 1.5$ & 158 \\
\hline D & 054145.3 & -015347 & 2011 Oct 18 & $0.03-0.07$ & 1.4 & $1.8 \times 1.5$ & 15 \\
\hline $\mathrm{E}$ & 054143.5 & -015330 & 2011 Oct 18 & $0.03-0.07$ & 1.0 & $1.8 \times 1.5$ & 15 \\
\hline $\mathrm{F}$ & 054143.2 & -015404 & 2011 Oct 18 & $0.03-0.07$ & 4.6 & $1.8 \times 1.5$ & 15 \\
\hline G & 054141.2 & -015340 & 2011 Dec 29 & $0.08-0.15$ & 2.2 & $2.2 \times 1.7$ & 5 \\
\hline $\mathrm{H}$ & 054139.2 & -015402 & 2011 Dec 29 & $0.08-0.15$ & 1.0 & $2.2 \times 1.7$ & 5 \\
\hline I & 054138.7 & -015323 & 2011 Dec 29 & $0.08-0.15$ & 1.4 & $2.2 \times 1.7$ & 5 \\
\hline
\end{tabular}

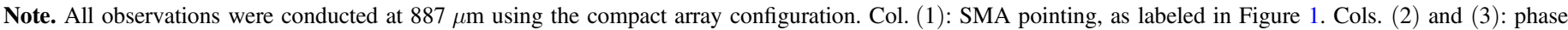

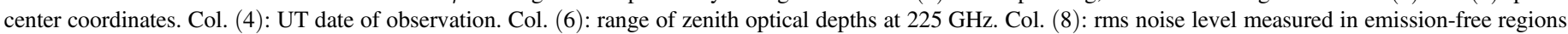
within the primary beam of the naturally weighted synthesized maps. Cols. (9) and (10): dimensions and orientations of the synthesized beams.
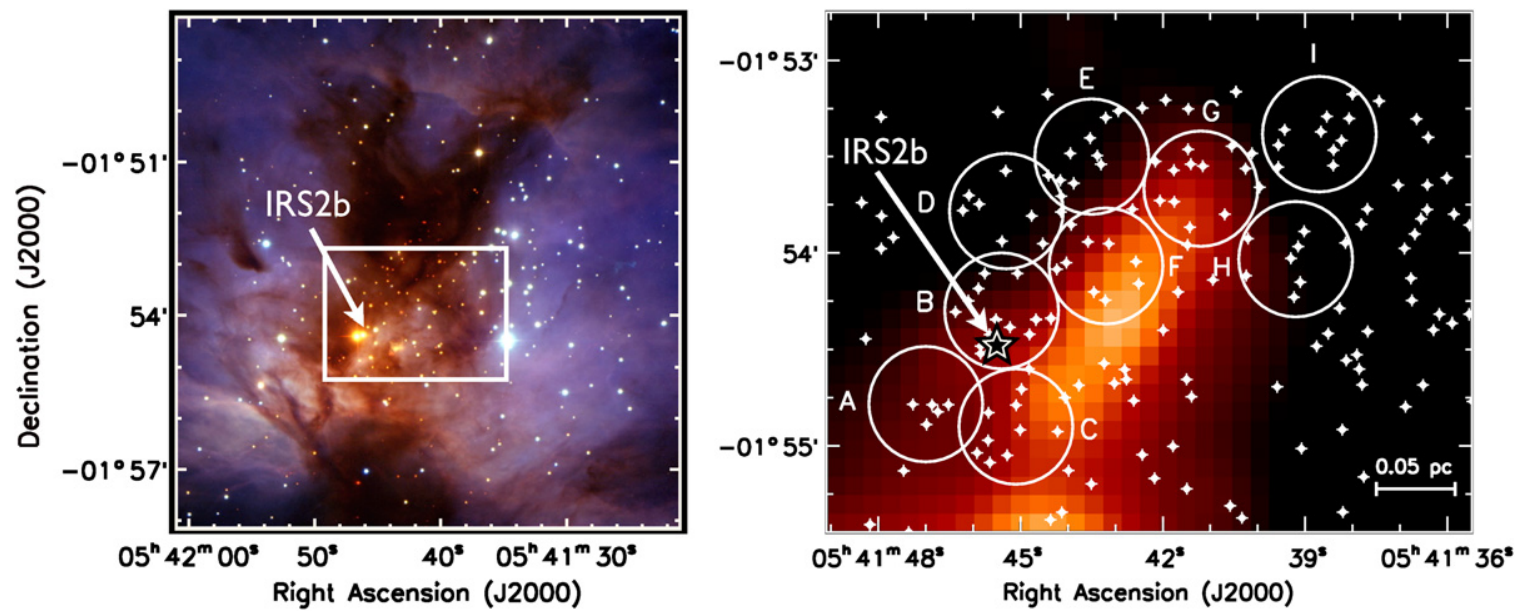

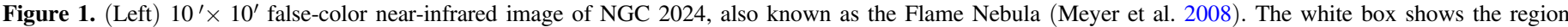

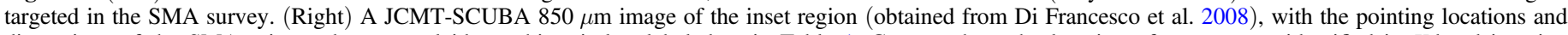

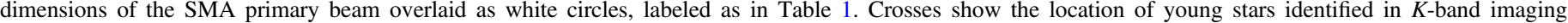
(Meyer 1996). The most massive star of the cluster, IRS 2b, is labeled in both panels.

unexplored. Eisner \& Carpenter (2003) made the sole attempt at measuring the NGC 2024 disk mass distribution, using a $3 \mathrm{~mm}$ survey of 150 targets with modest sensitivity (a disk mass upper limit of $\left.\sim 0.035 M_{\odot}\right)$. They detected two massive disks $\left(0.08\right.$ and $0.24 M_{\odot}$; significantly larger than seen in the ONC), and argued that image stacking suggested that the average disk mass was $\sim 0.005 M_{\odot}$ (comparable to TaurusAuriga and $\rho$ Ophiuchus).

Here we present the results of a new Submillimeter Array (SMA) survey of the $887 \mu \mathrm{m}$ continuum emission toward 95 young stars in the NGC 2024 cluster. Leveraging the steep scaling between the continuum flux and observing frequency $\left(F_{\nu} \propto \nu^{2-4}\right)$, these observations represent an order of magnitude improvement in sensitivity over the previous work in this region, and should be capable of detecting the average disk mass as suggested by Eisner \& Carpenter (2003). This survey represents the deepest attempt to measure the disk mass distribution in a very young, rich cluster, and thereby to probe how environment impacts basic disk properties. The observations and their calibration are described in Section 2. The flux measurements and their estimated conversion to disk masses are presented in Section 3. We make a comparison of the derived disk mass distribution with other regions, examine the dependence of disk mass on location in the cluster, and discuss the implications for planet formation in rich clusters in Section 4.

\section{OBSERVATIONS}

Observations of 9 distinct pointings containing a total of 95 young stars were conducted with the SMA (Ho et al. 2004) in the fall of 2011, using the compact array configuration (baselines of $\sim 8-50 \mathrm{~m}$ ). The pointing centers are listed in Table 1 and shown in Figure 1, and were chosen to maximize the number of young stars imaged in each ( $35^{\prime \prime}$ FWHM) primary beam while minimizing contamination from the bright, non-uniform molecular cloud background in the region (see Section 3). The SMA double sideband receivers were tuned to a local oscillator (LO) frequency of $338.213 \mathrm{GHz}(887 \mu \mathrm{m}$, see Table 1). Each sideband provided $4 \mathrm{GHz}$ of bandwidth, centered $\pm 5 \mathrm{GHz}$ from the LO frequency. Three observing tracks were shared between three separate pointings in each. The observations of the NGC 2024 fields were interleaved with nearby gain calibrators on 15 minute intervals. Weather conditions for all observations were good, with $<2 \mathrm{~mm}$ of precipitable water vapor, $\tau(225 \mathrm{GHz})<0.1$. Table 1 summarizes the relevant observational information. 


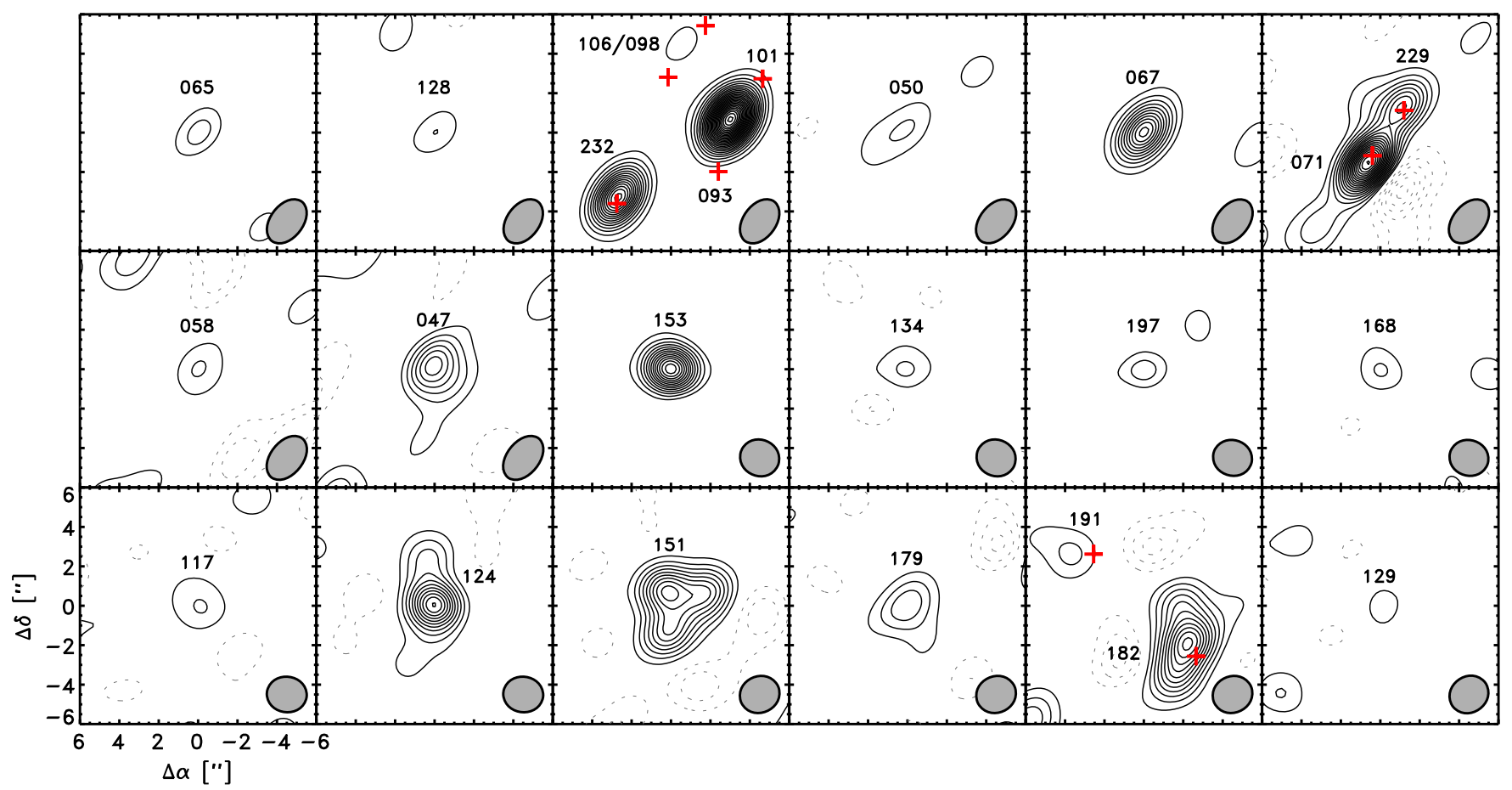

Figure 2. Synthesized images of the $887 \mu$ m continuum emission toward the 22 detected infrared sources (see Table 2). Each panel is $12^{\prime \prime}$ ( $5000 \mathrm{AU}$ ) on a side, and includes labels in the Meyer (1996) IRC designation. Contours are drawn at $3 \sigma$ intervals, and the synthesized beam dimensions are shown in the bottom right corners of each panel. In all but three panels, the image center corresponds to the infrared source position; otherwise, those positions are marked with crosses. The third panel includes the two cases with multiple possible identifications; see Table 2.

The raw visibilities were calibrated using the MIR software package. Passband calibration was conducted using the bright, compact radio sources, 3C 279 , 3C 84 , or J0854+201. The absolute flux scale was derived from observations of Titan and Uranus (and checked against 3C 111), and is accurate to $\sim 10 \%$. Amplitude and phase calibration were performed using observations of the nearby sources J0423-013, J0530+135, and J0607-085. The calibrated visibilities were naturally weighted and Fourier inverted, then CLEANed to generate the synthesized continuum maps shown in Figure 2 using MIRIAD (Sault et al. 1995). The synthesized maps were created after eliminating projected antenna spacings shorter than $27 \mathrm{k} \lambda$, to filter out extended emission on size scales $\geqslant 7 ! 15$. This scale was chosen to preserve the compact emission from the disks, while minimizing contamination from the bright, extended cloud background; it is the same as used in the analysis of dust emission from disks in the ONC (Mann \& Williams 2010) for the sake of consistency. Simulations of the background (see Section 3) confirmed that the $27 \mathrm{k} \lambda$ cutoff resolves out most of the extended emission and thereby helps reduce the effective rms noise levels by roughly a factor of $\sim 2$.

\section{RESULTS}

Continuum emission was detected toward 22 near-infrared sources at $>3 \times$ the measured rms noise level (see Table 2). ${ }^{7}$ The observed flux density, $F_{\mathrm{obs}}$, for each source was determined with a Gaussian fit in the image plane, after

\footnotetext{
7 An additional $\sim 20$ emission peaks (each at $\sim 3 \sigma$ ) were identified, but are not coincident with any known near-infrared sources (some of them are visible in Figure 2). This is the expected number of $3 \sigma$ noise peaks over such a large survey area (perhaps even an underestimate when considering the sparse Fourier sampling), although we cannot rule out the possibility that some are real sources associated with deeply embedded objects.
}

correcting for primary beam attenuation. The detected sources were associated with their stellar counterparts by reference to the near-infrared catalog of Meyer (1996). In two cases, IRC 106/098 and IRC 101/093 (see Table 2), it is difficult to unambiguously distinguish two potential associations with infrared sources. For the former, observations with improved sensitivity and resolution (and ideally Fourier sampling) will be required to robustly differentiate the options. On the other hand, it is entirely possible that the very bright emission near IRC 101/093 is unassociated with either source, but instead is tracing an embedded source at an earlier evolutionary stage. Another 73 infrared sources were covered in the survey, but not detected. Limits on their submillimeter continuum flux densities were determined from local measurements of the rms noise level, and are also listed in Table 2.

The observed flux densities are the linear combination of several emission contributors; free-free radiation from ionized material $\left(F_{\mathrm{ff}}\right)$, and thermal radiation from dust in the surrounding molecular cloud $\left(F_{\text {cloud }}\right)$ and the disk $\left(F_{\text {disk }}\right)$. We assumed that any free-free emission is optically thin, with a spectrum $F_{\mathrm{ff}} \propto \nu^{-0.1}$, and used deep VLA $3.6 \mathrm{~cm}$ measurements (Rodríguez et al. 2003, see Table 2) when available as normalizations to extrapolate $F_{\mathrm{ff}}$ up to $887 \mu \mathrm{m}$. Only four of the VLA $3.6 \mathrm{~cm}$ sources overlap with the SMA $887 \mu \mathrm{m}$ detections. Upper limits at $3.6 \mathrm{~cm}$ are sufficiently low $(\sim 50 \mu \mathrm{Jy}$ at $3 \sigma$ ) that extrapolated estimates of free-free contamination for the other targets are considered negligible. The observed targets are embedded in their host cloud, which itself produces significant dust emission on large spatial scales. To estimate that cloud contribution at each location in the SMA maps, we simulate the SMA response to its large-scale emission as observed with the SCUBA instrument on the James Clerk Maxwell Telescope (JCMT; Di Francesco et al. 2008), 
Table 2

Inferred Disk Fluxes and Masses

\begin{tabular}{|c|c|c|c|c|c|c|c|c|}
\hline $\begin{array}{l}\text { Source } \\
\text { (IRC) } \\
(1)\end{array}$ & (2) & (3) & $\delta(\mathbf{J} 2000)$ & $\begin{array}{c}F_{\mathrm{obs}} \\
(\mathrm{mJy}) \\
(5)\end{array}$ & $\begin{array}{c}F_{\mathrm{ff}} \\
(\mathrm{mJy}) \\
(6)\end{array}$ & $\begin{array}{c}F_{\text {cloud }} \\
(\mathrm{mJy}) \\
(7)\end{array}$ & $\begin{array}{c}F_{\text {disk }} \\
(\mathrm{mJy}) \\
(8)\end{array}$ & $\begin{array}{c}M_{\text {disk }} \\
\left(0.01 M_{\odot}\right) \\
(9)\end{array}$ \\
\hline $106 / 098$ & B & 054145.6 & -015422.4 & $10.7 \pm 3.0$ & $\cdots$ & 0.3 & 10.5 & $0.6 \pm 0.2$ \\
\hline 101/093* & B & 054145.4 & -015426.3 & $298.6 \pm 3.0$ & 0.7 & 0.3 & 297.7 & $16.0 \pm 0.2$ \\
\hline $232 *($ IRS 2$)$ & B & 054145.8 & -015430.2 & $209.4 \pm 3.0$ & 12.0 & 0 & 199.3 & $4.0 \pm 0.2$ \\
\hline $071^{*}$ & $\mathrm{C}$ & 054144.1 & -015445.8 & $330.5 \pm 3.0$ & 0.2 & 0.1 & 330.1 & $17.7 \pm 0.3$ \\
\hline 229 & $\mathrm{C}$ & 054144.0 & -015443.1 & $192.6 \pm 3.0$ & $\ldots$ & 0.2 & 192.5 & $10.3 \pm 0.4$ \\
\hline 058 & $\mathrm{C}$ & 054144.1 & -015454.6 & $34.1 \pm 3.0$ & $\ldots$ & 0.1 & 34.0 & $1.8 \pm 0.3$ \\
\hline 047 & $\mathrm{C}$ & 054144.1 & -015506.5 & $210.7 \pm 3.0$ & $\ldots$ & 0.1 & 210.6 & $11.3 \pm 0.4$ \\
\hline 153 & $\mathrm{D}$ & 054144.7 & -015348.7 & $55.0 \pm 1.2$ & $\ldots$ & 0 & 55.2 & $3.0 \pm 0.1$ \\
\hline 134 & D & 054144.7 & -015401.6 & $17.1 \pm 1.2$ & $\cdots$ & 0.1 & 17.0 & $0.9 \pm 0.1$ \\
\hline 151 & G & 054141.5 & -015348.6 & $179.5 \pm 2.0$ & $\ldots$ & 0.1 & 179.4 & $9.6 \pm 0.1$ \\
\hline 179 & $\mathrm{G}$ & 054141.7 & -015335.2 & $46.3 \pm 2.0$ & $\ldots$ & 0.0 & 46.4 & $2.5 \pm 0.1$ \\
\hline 191 & $\mathrm{G}$ & 054141.5 & -015326.7 & $32.3 \pm 2.0$ & $\ldots$ & 0 & 33.1 & $1.8 \pm 0.2$ \\
\hline 182 & $\mathrm{G}$ & 054141.1 & -015331.2 & $172.3 \pm 2.0$ & $\ldots$ & 0 & 172.7 & $9.3 \pm 0.1$ \\
\hline 129 & $\mathrm{H}$ & 054140.2 & -015406.6 & $6.3 \pm 1.3$ & $\ldots$ & 0 & 6.3 & $0.3 \pm 0.1$ \\
\hline
\end{tabular}

\begin{tabular}{|c|c|c|c|c|c|c|c|c|}
\hline $\begin{array}{l}\text { Non Detections } \\
066\end{array}$ & \multicolumn{8}{|c|}{$3 \sigma$ Upp Limit } \\
\hline 068 & A & 054146.7 & -015445.1 & 3.8 & $\cdots$ & 0.2 & 3.6 & 0.2 \\
\hline 062 & A & 054146.8 & -015449.3 & 3.8 & $\ldots$ & 0.2 & 3.6 & 0.2 \\
\hline 060 & $\mathrm{~A}$ & 054147.0 & -015453.1 & 3.9 & $\ldots$ & 0.3 & 3.6 & 0.2 \\
\hline 064 & A & 054147.3 & -015447.1 & 3.7 & $\cdots$ & 0.1 & 3.6 & 0.2 \\
\hline 107 & B & 054144.7 & -015420.7 & 12.0 & $\cdots$ & 0.2 & 11.8 & 0.6 \\
\hline 231 & $\mathrm{~B}$ & 054144.7 & -015431.4 & 17.1 & $\cdots$ & 0.2 & 16.9 & 0.9 \\
\hline 102 & $\mathrm{~B}$ & 054145.2 & -015423.1 & 9.7 & $\ldots$ & 0.2 & 9.5 & 0.5 \\
\hline 127 & B & 054145.7 & -015406.5 & 12.7 & $\ldots$ & 0.1 & 12.6 & 0.7 \\
\hline 122 & $\mathrm{~B}$ & 054145.9 & -015411.3 & 11.0 & 0.1 & 0.2 & 10.7 & 0.6 \\
\hline 114 & $\mathrm{~B}$ & 054146.1 & -015415.0 & 11.5 & $\ldots$ & 0.1 & 11.4 & 0.6 \\
\hline 109 & $\mathrm{~B}$ & 054146.4 & -015418.4 & 14.2 & $\cdots$ & 0.1 & 14.0 & 0.8 \\
\hline 073 & $\mathrm{C}$ & 054144.9 & -015442.6 & 12.0 & $\cdots$ & 0.1 & 11.9 & 0.6 \\
\hline 053 & $\mathrm{C}$ & 054145.9 & -015502.2 & 11.3 & $\cdots$ & 0.2 & 14.2 & 0.8 \\
\hline 157 & $\mathrm{D}$ & 054144.1 & -015347.5 & 7.1 & $\ldots$ & 0 & 7.1 & 0.4 \\
\hline 175 & $\mathrm{D}$ & 054144.4 & -015336.2 & 7.0 & $\ldots$ & 0.1 & 6.9 & 0.4 \\
\hline 138 & $\mathrm{D}$ & 054144.5 & -015357.4 & 6.2 & $\ldots$ & 0 & 6.2 & 0.3 \\
\hline 176 & $\mathrm{D}$ & 054145.3 & -015334.8 & 4.9 & $\ldots$ & 0.2 & 4.7 & 0.3 \\
\hline 139 & $\mathrm{D}$ & 054145.4 & -015356.5 & 4.4 & $\ldots$ & 0 & 4.4 & 0.2 \\
\hline 161 & $\mathrm{D}$ & 054145.9 & -015345.1 & 4.2 & $\ldots$ & 0.1 & 4.1 & 0.2 \\
\hline 167 & $\mathrm{D}$ & 054146.1 & -015342.3 & 5.0 & $\cdots$ & 0.1 & 4.9 & 0.3 \\
\hline 156 & $\mathrm{D}$ & 054146.2 & -015347.0 & 5.4 & $\ldots$ & 0.1 & 5.3 & 0.3 \\
\hline 206 & $\mathrm{E}$ & 054142.9 & -015316.3 & 5.4 & $\ldots$ & 0.1 & 5.3 & 0.3 \\
\hline 202 & $\mathrm{E}$ & 054143.2 & -015318.4 & 4.3 & $\ldots$ & 0.2 & 4.1 & 0.2 \\
\hline 181 & $\mathrm{E}$ & 054143.3 & -015332.8 & 3.1 & $\ldots$ & 0.2 & 2.9 & 0.2 \\
\hline 187 & $\mathrm{E}$ & 054143.3 & -015330.2 & 3.0 & $\ldots$ & 0.2 & 2.8 & 0.2 \\
\hline
\end{tabular}


Table 2

(Continued)

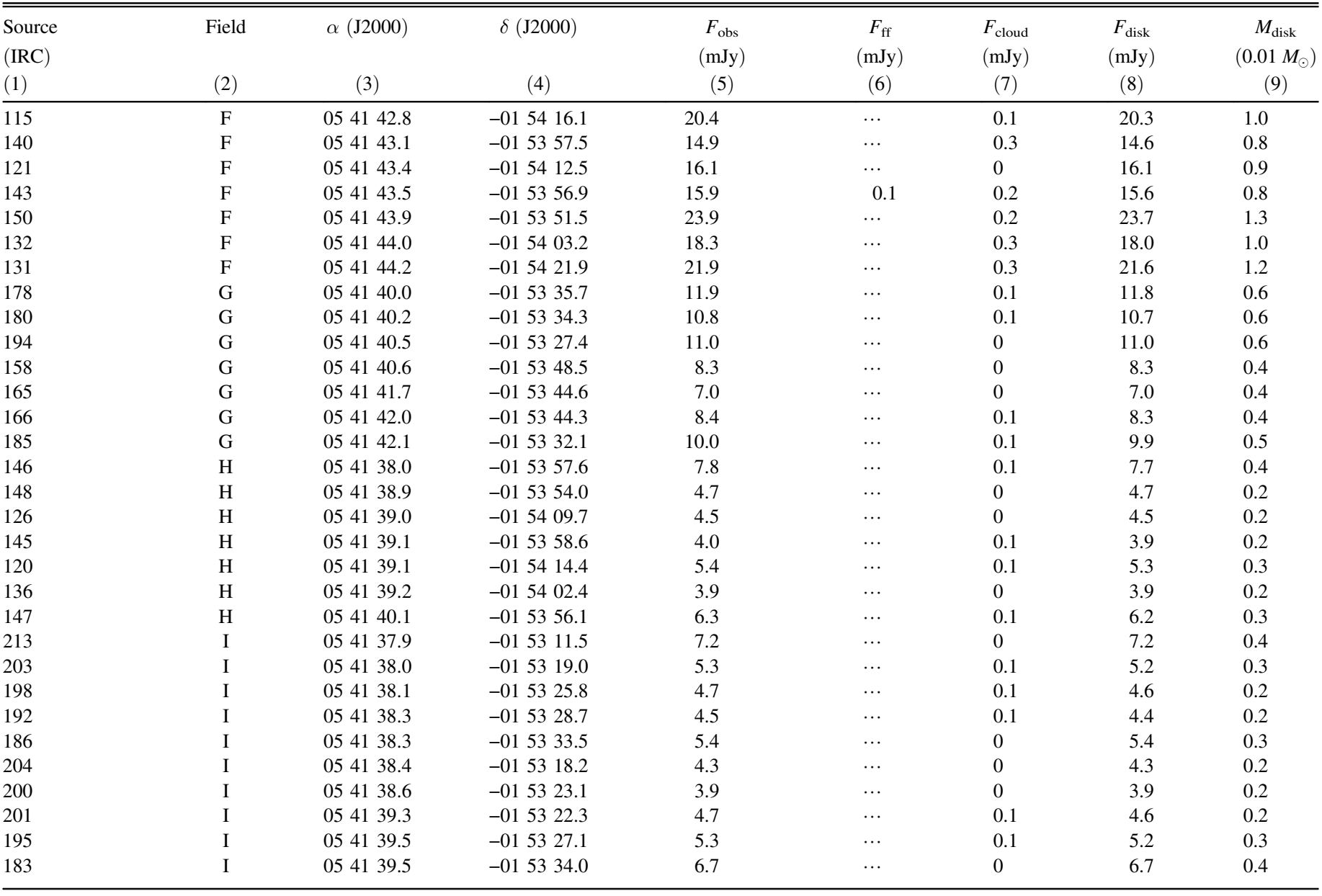

Note. Sources associated with asterisks were also detected at $3 \mathrm{~mm}$ by Eisner $\&$ Carpenter (2003): IRC 106/098 $=$ source 8 , IRC $232=$ source 9 (IRS 2 ), IRC $071=$ source 4, and IRC $124=$ source 1. Col. (1): source designation, according to Meyer (1996). Col. (2): SMA field, as labeled in Figure 1 and Table 1. Cols. $(3,4)$ : SMA emission centroid coordinates. Col. (5): Integrated continuum flux density, or $3 \sigma$ upper limits on the non-detections, corrected for SMA primary beam attenuation. Col. (6): extrapolated contribution of free-free emission at $887 \mu \mathrm{m}$ estimated from Rodríguez et al. (2003) measurements. Col. (7): estimated contribution from large-scale cloud emission. Col. (8): derived dust continuum flux density from the disk. Col. (9): inferred disk mass (uncertainty does not include systematics in the absolute flux scale, which contribute an additional $\sim 10 \%$ ).

following the approach of Mann \& Williams (2010). For each SMA pointing, we Fourier transformed the appropriate JCMT map, sampled the emission onto the observed $u, v$-tracks, and generated CLEANed maps. The cloud emission, $F_{\text {cloud }}$, was then estimated toward each source location. We found that cloud contamination was typically $<10 \%$, considerably lower than in the ONC. After accounting for any free-free or cloud contamination, the remainder of $F_{\text {obs }}$ was associated with the disk, $F_{\text {disk. }}$. Table 2 lists the decomposed contributions for each source.

Relatively little is known about the nature of the infrared sources associated with submillimeter emission in this survey. Given their neutral or modestly red $J H K$ colors, we are making the assumption that the vast majority of them are in the socalled Class II or T Tauri stage, young stars with disks but no remnant envelope material. It is possible that the few most luminous sources could be in the earlier Class I stage, if their observed near-infrared emission is primarily tracing scattered light from outflow cavities in their envelope structures (Eisner 2012; Sheehan \& Eisner 2014). As we noted above, the very brightest source in this sample cannot be unambiguously associated with an infrared source; it is plausible that the emission may originate in a dense envelope around a Class 0 protostar. Without a more complete set of ancillary information (e.g., full spectral energy distributions), a refined classification is not yet feasible.

Following Eisner \& Carpenter (2003), we aimed to constrain the mean emission level of the 73 undetected sources in the SMA fields by "stacking" the data. However, there is sufficient concern with combining data in the image plane when the individual fields were sampled so sparsely in the Fourier domain. Instead we performed a complementary analysis on the visibilities. First, we removed the detected sources by subtracting Gaussian models of the emission from the observed visibilities. We then generated 73 permutations of the visibility data, each with a phase shift that accounts for the location of the undetected cluster member. ${ }^{8}$ Those permutations were coadded and then imaged as described in Section 2. We found no

\footnotetext{
8 Technically this includes substantial duplication of the data. However, the individual sources are located far enough apart that their mutual contributions at any given phase shift are considered negligible.
} 
emission associated with this stacked data set, and placed a $3 \sigma$ upper limit of $\sim 2.4 \mathrm{mJy}$ on the ensemble average. Assuming a typical spectrum that scales like $F_{\nu} \propto \nu^{2-3}$, this limit is 4-10x lower than the ensemble mean flux density that was estimated from image-plane stacking by Eisner \& Carpenter (2003).

We assume that the observed continuum emission is (mostly) optically thin, and therefore a sensitive probe of the dust mass. Since most of the emission originates in the cool, nearly isothermal outer regions of a disk, we estimate the mass as

$$
M_{\mathrm{disk}}=\frac{d^{2} F_{\mathrm{disk}}}{\kappa_{\nu} B_{\nu}(T)},
$$

where $d$ is the distance, $\kappa_{\nu}$ is the opacity per gram of the disk material, and $B_{\nu}(T)$ is the Planck function at a characteristic temperature (e.g., Beckwith et al. 1990). Disk masses were calculated for the 22 detected sources (see Table 2) using Equation (1) and standard assumptions (for ease of comparison with other studies): a characteristic dust temperature $T=20 \mathrm{~K}$, the Beckwith et al. (1990) opacity $\kappa_{\nu}=0.034 \mathrm{~cm}^{2} \mathrm{~g}^{-1}$ at $887 \mu \mathrm{m}$ (which implicitly assumes a 100:1 gas-to-dust mass ratio), and a distance $d=415 \mathrm{pc}$ to NGC 2024, based on observations of B-type stars in the cluster (AnthonyTwarog 1982). Disk emission was inferred toward the source IRS 2 (IRC 232), an early B-type star; in calculating its mass, we adopted a higher dust temperature of $40 \mathrm{~K}$ (e.g., see Beuther et al. 2002; Sridharan et al. 2002). Systematic uncertainties in the $M_{\text {disk }}$ estimates are dominated by the poorly constrained values of the dust opacities, which are ambiguous at the order of magnitude level (e.g., Henning \& Stognienko 1996). Some relatively minor additional uncertainties from optical depth effects could also contribute, especially given the unknown disk structures: Andrews \& Williams (2005) estimated that these are on the order of $\sim 10 \%$ for the fainter luminosities that characterize this sample, but could rise to as high as $\sim 50 \%$ at the high luminosity end.

The overall disk mass sensitivity of this SMA survey depends on the locations of each target relative to the field (pointing) center, the varying levels of cloud emission, and any free-free emission contributions. A mass completeness level for the survey was estimated based on Monte Carlo simulations. Synthetic disks (point sources) with emission appropriate for a given $M_{\text {disk }}$ (see Equation (1)) were injected into the large-scale emission maps from the JCMT, Fourier inverted and sampled onto the observed spatial frequencies, and then each field was imaged as in Section 2. By measuring the fraction of synthetic targets that were detected $(>3 \sigma)$ for each input $M_{\text {disk }}$ in these Monte Carlo simulations, we determined that the survey is essentially $100 \%$ complete for $M_{\text {disk }} \geqslant 0.01$ $M_{\odot}$, and roughly $50 \%$ complete for $M_{\text {disk }} \geqslant 0.004 M_{\odot}$. The upper limit on the stacked ensemble of undetected sources described above corresponds to $\sim 0.001 M_{\odot}$.

Four of the 13 sources detected by Eisner \& Carpenter (2003) at $3 \mathrm{~mm}$ were also detected in the SMA survey at $887 \mu \mathrm{m}$ : IRC 124, IRC 071, IRC 101, and IRS 2 (their sources $1,4,8$, and 9 , respectively). The remaining nine sources were either outside our survey area (their sources 3, 11, 12, and 13) or were not detected with the SMA (IDs 2, 5, 6, 7, 9, and 10); the latter cases likely indicate that the radiation detected at $3 \mathrm{~mm}$ was generated by a non-dust emission mechanism. The observed flux ratios can be used to constrain the spectral slopes between $887 \mu \mathrm{m}$ and $3 \mathrm{~mm}$ of the targets with overlapping

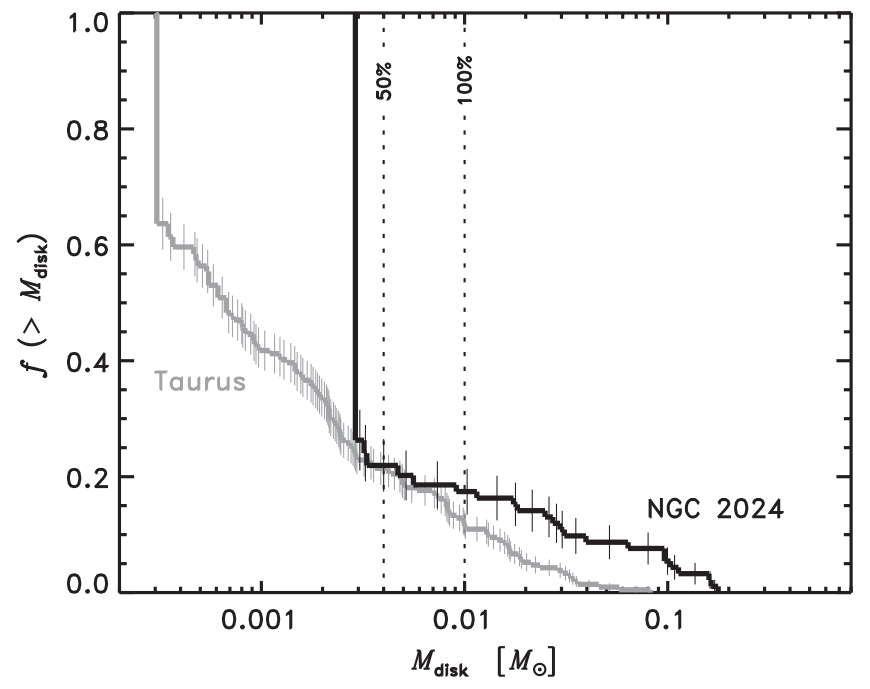

Figure 3. Cumulative distribution of disk masses in the NGC 2024 survey, constructed with the Kaplan-Meier product limit estimator to account for upper limits. The estimated 50 and 100\% completeness levels are marked as dotted vertical lines. The analogous distribution for the Taurus star-forming region (Andrews et al. 2013) is shown in gray for reference. The upper end of the NGC 2024 disk mass distribution favors slightly higher masses, as might be expected for a younger cluster, although the selection effects of this sample are not yet well characterized.

detections. We find spectral indices, $\alpha$ where $F_{\nu} \propto \nu^{\alpha}$, of $2.8 \pm 0.2($ IRC $124=$ source 1$), 2.8 \pm 0.1($ IRC $071=$ source 4), $2.6 \pm 0.1 \quad($ IRC $101=$ source 8$)$, and $0.1 \pm 0.1$ (IRS $2=$ source 9); the IRS 2 measurement is obviously strongly impacted by free-free contamination at $3 \mathrm{~mm}$. These spectral indices are at the higher end of the distributions inferred in $1-3$ Myr-old star-forming clusters (Ricci et al. 2010a, 2010b, 2011a, 2011b), perhaps hinting at some age evolution in the disk-integrated opacity spectrum due to dust grain growth (e.g., Miyake \& Nakagawa 1993; Draine 2006). More measurements for young sources in NGC 2024 would be desirable.

\section{DISCUSSION}

We conducted a large-scale survey of the $887 \mu \mathrm{m}$ continuum emission toward 95 young stars in the 0.3 Myr-old NGC 2024 cluster using the SMA. Assuming standard conversions for optically thin dust emission, this survey is complete down to a disk mass limit of $\sim 0.01 M_{\odot}(3 \sigma)$, although in some regions the survey is slightly more sensitive. We detected a total of 22 disks $(23 \pm 5 \%$ of the sample), including 4 that were previously detected at $3 \mathrm{~mm}$ by Eisner \& Carpenter (2003).

Figure 3 shows the cumulative distribution of disk masses in this survey, where we have incorporated the upper limits by employing the Kaplan-Meier product limit estimator for a censored sample (Feigelson \& Nelson 1985). Although these data are sensitivity-limited to probe only relatively massive disks, we find that the fraction of disks with large $M_{\text {disk }}$ is relatively high: $\sim 20 \%$ have $M_{\text {disk }}>0.01 M_{\odot}$, and $\sim 10 \%$ have $M_{\text {disk }}>0.1 M_{\odot}$. Taken at face value, this suggests that the highmass tail of the NGC $2024 M_{\text {disk }}$ distribution is more populated than in slightly older ( 1-3 Myr) clusters like the ONC (Mann et al. 2014), Ophiuchus (Andrews \& Williams 2007), and Taurus (see the corresponding distribution function in Figure 3 for a direct comparison; Andrews \& Williams 2005; Andrews 


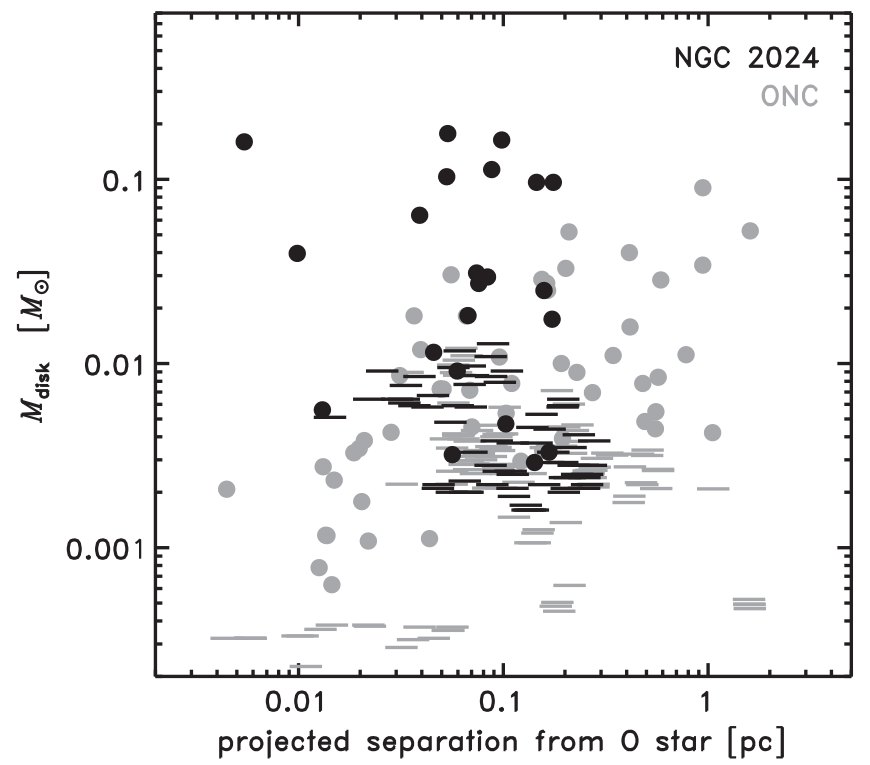

Figure 4. Disk masses in the NGC 2024 (black) and ONC (gray; see Mann \& Williams 2010; Mann et al. 2014) clusters as a function of their projected separations from the nearest massive star, IRS $2 \mathrm{~b}$ and $\theta^{1}$ Ori $\mathrm{C}$, respectively. Circles represent submillimeter continuum detections of dust disk emission, and horizontal line segments mark $3 \sigma$ upper limits. The depletion in $M_{\text {disk }}$ at small projected separations seen for the ONC is not apparent for the NGC 2024 cluster, perhaps because it represents an earlier evolutionary stage or due to the (presumably) comparatively weaker photoionizing radiation field present.

et al. 2013). The standard censored two-sample tests advocated by Feigelson \& Nelson (1985) indicate a marginal $(\sim 2 \sigma)$ quantitative offset, with the NGC 2024 distribution shifted to higher masses by a factor of $\sim 1.5-2$.

However, such comparisons can be misleading if they do not account for selection biases. Disk masses are known to depend on factors like the (host) mass (Andrews et al. 2013), multiplicity (Harris et al. 2012; Akeson \& Jensen 2014), and evolutionary state (Andrews \& Williams 2005) of the target. Unfortunately, little is known about these properties for the NGC 2024 sample. If we make the assumptions that this survey has targets drawn from the same host mass function and with the same multiplicity statistics as in Taurus, and suggest that all of the near-infrared sources in the SMA fields harbor disks with no envelopes, we can use the Monte Carlo approach advocated by Andrews et al. (2013) to compare with the reference $M_{\text {disk }}$ distribution in Taurus. The results indicate that the two $M_{\text {disk }}$ distributions are statistically indistinguishable (given the current data), suggesting that there is relatively little evolution in $M_{\text {disk }}$ up to a few Myr. More robust constraints on changes in this distribution would require a better characterization of the NGC 2024 targets, and secondarily an expanded continuum census.

But independent of these (potential) selection effects, a particularly interesting comparison can still be made between the disks in the NGC 2024 cluster and the ONC, since both regions host high-mass stars in their immediate environments that could potentially modify the disk mass distribution. Sources in the ONC found within $\sim 0.03 \mathrm{pc}$ of the massive star $\theta^{1}$ Ori $\mathrm{C}$ have systematically lower $M_{\text {disk }}$ than those at larger separations (Mann \& Williams 2009, 2010; Mann et al. 2014), reflecting the consequences of external evaporation from strong photoionizing sources on disk dissipation timescales (Johnstone et al. 1998; Störzer and Hollenbach 1999; Richling \&
Yorke 2000; Scally \& Clarke 2001; Matsuyama et al. 2003; Adams et al. 2004). Figure 4 shows $M_{\text {disk }}$ as a function of the projected distance from the most massive stars in both the NGC 2024 cluster and the ONC, IRS $2 \mathrm{~b}$ and $\theta^{1}$ Ori C, respectively. Unlike the $\mathrm{ONC}$, we find no evidence of a distance-dependent disk mass distribution in NGC 2024. Although the total number of disk detections in the NGC 2024 region is limited, several massive disks identified here are located $<0.01 \mathrm{pc}$ from IRS $2 \mathrm{~b}$.

We consider two likely, and not mutually exclusive, reasons for this difference between NGC 2024 and the ONC. First is an evolutionary argument, based on the fact that NGC 2024 appears to be considerably younger than the ONC (see Meyer 1996; Eisner \& Carpenter 2003). That relative youth could mean that we are observing more of a primordial $M_{\text {disk }}$ distribution in NGC 2024, before external evolutionary processes like photoevaporation have had time to make a significant impact. Second is an environmental distinction, in that IRS $2 b$ is an intrinsically less luminous source than $\theta^{1}$ Ori $\mathrm{C}$, and therefore could produce a substantially weaker photoionizing radiation field that is less capable of stripping material from its surrounding disks. Bik et al. (2003) suggested that IRS $2 \mathrm{~b}$ has a spectral type of O8-B2, although the radio continuum flux measured by Barnes et al. (1989) indicates that the earlier type is more appropriate. However, Meyer et al. (1997) instead suggested that early B-types are the preferred spectroscopic classification for IRS $2 b$, and that it may not be the sole or even dominant source of ionizing radiation in the region (see also Meyer et al. 2008). A small group of weaker ionizing sources might provide sufficiently attenuated massloss rates and explain the absence of a separation dependence on the $M_{\text {disk }}$ distribution. Even if IRS $2 \mathrm{~b}$ was more luminous than described, NGC 2024 is more heavily extincted than the $\mathrm{ONC}$, and the high energy photons could be easily absorbed by the cloud.

Future, more sensitive observations of NGC 2024 with the Atacama Large Millimeter Array will permit an exploration of the full disk mass distribution in this young region, facilitating stronger constraints on disk dissipation by ultraviolet photoevaporation, on the evolutionary timescales of disks in rich clusters, and on the initial conditions of the planet formation process for the majority of stars in the galaxy. Such campaigns should necessarily be coupled with a more comprehensive characterization of the NGC 2024 stellar population.

We thank the referee for a very helpful review. The Submillimeter Array is a joint project between the Submillimeter Astrophysical Observatory and the Academica Sinica Institute of Astronomy and Astrophysics and is funded by the Smithsonian Institution and the Academica Sinica.

\section{REFERENCES}

Adams, F. C., Hollenbach, D., Laughlin, G., \& Gorti, U. 2004, ApJ, 611, 360 Akeson, R. L., \& Jensen, E. L. N. 2014, ApJ, 784, 62

Ali, B., Sellgren, K., Depoy, D. L., et al. 1998, ASP Conf. Ser. 154, Cool Stars, Stellar Systems, and the Sun, ed. R. A. Donahue, \& J. A. Bookbinder (San Francisco, CA: ASP), 1663

Andrews, S. M., Rosenfeld, K. A., Kraus, A. L., \& Wilner, D. J. 2013, ApJ, 771,129

Andrews, S. M., \& Williams, J. P. 2005, ApJ, 631, 1134

Andrews, S. M., \& Williams, J. P. 2007, ApJ, 671, 1800

Andrews, S. M., Wilner, D. J., Hughes, A. M., Qi, C., \& Dullemond, C. P. 2009, ApJ, 700, 1502

Andrews, S. M., Wilner, D. J., Hughes, A. M., Qi, C., \& Dullemond, C. P. 2010, ApJ, 723, 1241 
Anthony-Twarog, B. J. 1982, AJ, 87, 1213

Baraffe, I., Chabrier, G., Allard, F., \& Hauschildt, P. H. 1998, A\&A, 337, 403

Barnes, P. J., Crutcher, R. M., Bieging, J. H., Storey, J. W. V., \& Willner, S. P. 1989, ApJ, 342, 883

Beckwith, S. V. W., Sargent, A. I., Chini, R. S., \& Guesten, R. 1990, AJ, 99, 924

Beuther, H., Schilke, P., Menten, K. M., et al. 2002, ApJ, 566, 945

Bik, A., Lenorzer, A., Kaper, L., et al. 2003, A\&A, 404, 249

Bonnell, I. A., Bate, M. R., \& Vine, S. G. 2003, MNRAS, 343, 413

da Rio, N., Robberto, M., Soderblom, D. R., et al. 2010, ApJ, 722, 1092

D’Antona, F., \& Mazzitelli, I. 1997, MmSAI, 68, 807

di Francesco, J., Johnstone, D., Kirk, H., MacKenzie, T., \& Ledwosinska, E. 2008, ApJS, 175, 277

Draine, B. T. 2006, ApJ, 636, 1114

Eisner, J. A. 2012, ApJ, 755, 23

Eisner, J. A., \& Carpenter, J. M. 2003, ApJ, 598, 1341

Eisner, J. A., \& Carpenter, J. M. 2006, ApJ, 641, 1162

Eisner, J. A., Plambeck, R. L., Carpenter, J. M., Corder, S. A., Qi, C., \& Wilner, D. 2008, ApJ, 683, 304

Evans, N. J., II, Dunham, M. M., Jørgensen, J. K., et al. 2009, ApJS, 181, 321

Feigelson, E. D., \& Nelson, P. I. 1985, ApJ, 293, 192

Grasdalen, G. L. 1974, ApJ, 193, 373

Haisch, K. E., Jr., Lada, E. A., \& Lada, C. J. 2000, AJ, 120, 1396

Haisch, K. E., Jr., Lada, E. A., Piña, R. K., Telesco, C. M., \& Lada, C. J. 2001, AJ, 121, 1512

Harris, R. J., Andrews, S. M., Wilner, D. J., \& Kraus, A. L. 2012, ApJ, 751,115

Henning, T., \& Stognienko, R. 1996, A\&A, 311, 291

Ho, P. T. P., Moran, J. M., \& Lo, K. Y. 2004, ApJL, 616, L1

Johnstone, D., Hollenbach, D., \& Bally, J. 1998, ApJ, 499, 758

Lada, C. J. 1991, NATO ASIC Proc. 342, The Physics of Star Formation and Early Stellar Evolution, ed. C. J. Lada, \& N. D. Kylafis (Netherlands: Springer), 329

Lada, C. J., \& Lada, E. A. 2003, ARA\&A, 41, 57

Levine, J. L., Steinhauer, A., Elston, R. J., \& Lada, E. A. 2006, ApJ, 646, 1215
Mann, R. K., Di Francesco, J., Johnstone, D., et al. 2014, ApJ, 784, 82

Mann, R. K., \& Williams, J. P. 2009, ApJL, 694, L36

Mann, R. K., \& Williams, J. P. 2010, ApJ, 725, 430

Matsuyama, I., Johnstone, D., \& Hartmann, L. 2003, ApJ, 582, 893

Meyer, M. R. 1996, PhD thesis, Max-Planck-Institut für Astronomie

Meyer, M. R., Calvet, N., \& Hillenbrand, L. A. 1997, AJ, 114, 288

Meyer, M. R., Flaherty, K., Levine, J. L., et al. 2008, Star Formation in NGC 2023, NGC 2024, and Southern L1630, ed. B. Reipurth (San Francisco, CA: ASP), 662

Miyake, K., \& Nakagawa, Y. 1993, Icarus, 106, 20

Osterloh, M., \& Beckwith, S. V. W. 1995, ApJ, 439, 288

Porras, A., Christopher, M., \& Allen, L. 2003, AJ, 126, 1916

Reggiani, M., Robberto, M., da Rio, N., et al. 2011, A\&A, 534, A83

Ricci, L., Mann, R. K., Testi, L., et al. 2011a, A\&A, 525, A81

Ricci, L., Testi, L., Natta, A., \& Brooks, K. J. 2010a, A\&A, 521, A66

Ricci, L., Testi, L., Natta, A., et al. 2010b, A\&A, 512, A15

Ricci, L., Testi, L., Williams, J. P., Mann, R. K., \& Birnstiel, T. 2011b, ApJL, 739, L8

Richling, S., \& Yorke, H. W. 2000, ApJ, 539, 258

Rodríguez, L. F., Gómez, Y., \& Reipurth, B. 2003, ApJ, 598, 1100

Sault, R. J., Teuben, P. J., \& Wright, M. C. H. 1995, in ASP Conf. Ser. 77, Astronomical Data Analysis Software and Systems IV, ed. R. A. Shaw, H. E. Payne, \& J. J. E. Hayes (San Francisco, CA: ASP), 433

Scally, A., \& Clarke, C. 2001, MNRAS, 325, 449

Sheehan, P. D., \& Eisner, J. A. 2014, ApJ, 791, 19

Soderblom, D. R., Hillenbrand, L. A., Jeffries, R. D., Mamajek, E. E., \& Naylor, T. 2014, in Protostars and Planets VI, ed. H. Beuther et al. (Tucson, AZ: Univ. Arizona Press), 219

Sridharan, T. K., Beuther, H., Schilke, P., Menten, K. M., \& Wyrowski, F. 2002, ApJ, 566, 931

Störzer, H., \& Hollenbach, D. 1999, ApJ, 515, 669

Williams, J. 2010, ConPh, 51, 381

Williams, J. P., Andrews, S. M., \& Wilner, D. J. 2005, ApJ, 634, 495

Williams, J. P., Cieza, L. A., Andrews, S. M., et al. 2014, MNRAS, 435,1671 